\title{
Diffusion Tensor Tractography in Mesencephalic Bundles: Relation to Mental Flexibility in Detoxified Alcohol-Dependent Subjects
}

\author{
Sandra Chanraud ${ }^{1,2,3}$, Michel Reynaud4, Michèle Wessa ${ }^{1,2,3}$, Jani Penttilä' ${ }^{1,2,3}$, Nikoleta Kostogianni ${ }^{5}$, \\ Arnaud Cachia ${ }^{1,2,3}$, Eric Artiges ${ }^{1,2,3}$, Françoise Delain ${ }^{4}$, Murielle Perrin ${ }^{6}$, Henri-Jean Aubin ${ }^{5}$, \\ Yann Cointepas ${ }^{6}$, Catherine Martelli ${ }^{4}$ and Jean-Luc Martinot ${ }^{*, 1,2,3}$
}

'INSERM, U797 Research Unit 'Neuroimaging and Psychiatry', IFR49, Orsay, France; 'CEA, 'Neuroimaging and Psychiatry' U797 Unit, Hospital Department Frédéric Joliot \& Neurospin, I²BM, Orsay, France; ${ }^{3}$ Université Paris-Sud, UMR U797, Orsay and Université Paris 5 Rene Descartes, UMR U797, Paris, France; ${ }^{4}$ APHP, Department of Psychiatry and Addictology, Paul Brousse Hospital, Villejuif, France; ${ }^{5}$ APHP, Addiction Treatment Center, Emile Roux Hospital, Limeil-Brevannes, France; ${ }^{6}$ Image Analysis and Structural Anatomy Group, Neurospin, IFR 49, CEA, Gif-sur-Yvette, France

\begin{abstract}
Components of the corticocerebellar circuit and the midbrain individually play a central role in addictive processes and have been associated with altered volumes and impairment of cognitive flexibility in alcohol-dependent subjects. The microstructure of white matter bundles composing the corticocerebellar network and passing through the midbrain was studied using diffusion tensor imaging in a group of detoxified alcohol-dependent men $(n=20)$ and a group of healthy men $(n=24)$. The relationship between properties of these white matter bundles and cognitive flexibility performance was investigated in alcohol-dependent subjects. Bundles connecting two regions of interest were analyzed using a fiber-tracking quantitative approach, which provided estimates of the fractional anisotropy and the apparent diffusion coefficient, as well as the number of tracked fibers normalized by the volume of regions of interest. Within the bundles running between the midbrain and pons, a mean of $18 \%$ fewer fibers per unit volume were tracked in alcohol-dependent men than in healthy controls. In addition, the normalized number of these fibers correlated with the performance in the Trail-Making Test part-B. Even though the alcohol-dependent subjects were detoxified and apparently neurologically intact, their earlier excessive use of alcohol seems to be associated with altered neural microstructure of mesencephalic white matter bundles, which may contribute to their cognitive flexibility impairment.
\end{abstract}

Neuropsychopharmacology (2009) 34, 1223-1232; doi:I0. I038/npp.2008. I0 I; published online 9 July 2008

Keywords: alcohol; diffusion magnetic resonance imaging; brain stem; pons; thalamus; cognition

\section{INTRODUCTION}

In the corticocerebellar circuit, the midbrain is a core region that contains both top-down and bottom-up fiber bundles (Wakana et al, 2004), and it is highly involved in addictive processes (Hyman et al, 2006). Also, the pons and the thalamus have been identified as sites of an intermediate step in the feed-forward and feed-back loops of the corticocerebellar circuit, respectively (Schmahmann and Pandya, 1997). Chronic alcohol exposure has been associated with smaller volumes of the thalamus, pons, and cerebellum (Baker et al, 1999; Kril et al, 1997; Sullivan et al,

\footnotetext{
*Correspondence: Dr J-L Martinot, CEA-INSERM U797, Hospital Department Frédéric Joliot, 12BM, SHFJ-CEA. 4, place du Général Leclerc, 9|40 I Orsay. France, Tel: + 33 | 69867757,

Fax: + 33 I 6986 7810, E-mail: jean-luc.martinot@cea.fr Received 16 November 2007; revised 23 May 2008; accepted 23 May 2008
}

$2000,2003)$, as well as with cortical volume alterations (Harper et al, 2003).

Alcohol-dependent individuals often express distinct impairments in problem solving, cognitive planning, and working memory (Oscar-Berman et al, 1997). Such neuropsychological deficits have classically been associated with impaired function of the frontal lobes, but more recently, also with alterations within the corticocerebellar circuit (Schmahmann and Pandya, 1997; Sullivan, 2003). Previous investigations on alcoholism have provided evidence of an association between white matter structure in the corpus callosum and certain components of executive functions, eg attention skills (Pfefferbaum et al, 2000) and inhibitory control (Schulte et al, 2006). In our own recent study, decreased white matter volume in the midbrain of alcoholdependent subjects was related to impaired performance in two executive tasks: the Trail-Making Test part-B (TMT-B) and the WAIS letter-number sequencing test (LNS; Chanraud et al, 2007). Both tasks invoke cognitive flexibility, 
a component of executive functions, which is known to influence treatment outcome (Goldman, 1990) and experience-dependent recovery (Roehrich and Goldman, 1993) in chronic alcoholism. However, our earlier study did not identify the specific regions of the midbrain and associated white matter tracts that are involved in the alcoholdependent subjects' cognitive flexibility impairment.

Over the last decade, diffusion tensor imaging (DTI) has increasingly been applied to the assessment of white matter microstructure characteristics in normal development as well as various neurological conditions and psychiatric disorders. This noninvasive technique allows imaging of the microstructure of biological tissue (Le Bihan et al, 2001). In addition to quantitative fiber tracking (Johansen-Berg and Behrens, 2006; Sullivan et al, 2006), DTI can measure the apparent diffusion coefficient (ADC; a direction-independent measure of average diffusivity that reflects water motility in a voxel; see Le Bihan et al, 2001) and fractional anisotropy (FA; an estimate of highly restricted diffusion in a linear framework; see Pfefferbaum et al, 2006) in the white matter tissue.

In order to explore the yet largely unknown relationship between white matter characteristics in the corticocerebellar circuit and cognitive flexibility in alcohol-dependent subjects, we performed a tractography analysis of our DTI data (Chanraud et al, 2007). We hypothesized that microstructural alterations in white matter fiber bundles linking the midbrain to the pons and/or to the thalamus would be related to the impaired cognitive flexibility of these subjects, but not to other types of cognitive deficits (eg semantic memory) present in alcohol-dependent subjects.

\section{MATERIALS AND METHODS}

\section{Subjects}

DTI images from 20 alcohol-dependent subjects (DSM-IV criteria) and 24 healthy subjects were selected from a larger sample (Chanraud et al, 2007) on the basis of the DTI image availability. Data from one alcohol-dependent subject were excluded from the analysis to enable matching of groups for age and educational level (Table 1).

All study participants were Caucasian men, between 25 and 65 years of age, fluent in French, and right handed, as assessed by Annett's questionnaire (Annett, 1967). Patients were recruited on admission to detoxification or day-care units in two addiction departments of the Paul Brousse and Emile Roux hospitals in the Paris area (Assistance Publique, Hôpitaux de Paris, France).

All subjects were evaluated using the Alcohol Use Disorders Identification Test (AUDIT; Reinert and Allen, 2002) and the Social Adjustment Scale Self Report (Weissman and Bothwell, 1976; see Table 1).

Alcohol-dependent patients were interviewed and clinically examined by a senior psychiatrist (CM, JLM, or HJA). The medical consequences of chronic alcoholism on other organs may confound the association between brain microstructure and behavioral measures. Thus, as brain changes maybe, in part, secondary to liver damage in alcohol-dependent patients with alcoholic cirrhosis (Arria et al, 1991), we selected detoxified drinkers with moderate alcoholism, ie with no clinical evidence of brain dysfunction or medical conditions considered to be clinical indicators of severe alcoholism (eg alcohol-induced dementia or chronic liver disease). The inclusion criteria were (1) fewer than three periods of withdrawal because more than two periods of withdrawal may be associated with greater cognitive impairment in alcohol-dependent subjects (Duka et al, 2003), (2) detoxification for at least 3 weeks and abstinence, as assessed by normal levels of $\gamma$-glutamyl-transferase and carbohydrate-deficient transferrin (see Table 2), and (3) no use of sedative medications for at least 7 days preceding the study (before participating in this study, the patients had been treated during withdrawal with decreasing doses of lorazepam and vitamins B1 and B6). The exclusion criteria included (1) signs or symptoms of malnutrition, (2) signs of liver dysfunction: aspartate aminotransferase/alanine aminotransferase ratio greater than two (Cohen and

Table I Demographic Data and Neuropsychological Test Results of the Study Subjects

Alcohol-dependent subjects $(n=20) \quad$ Healthy subjects $(n=24) \quad$ Statistical significance*

\begin{tabular}{|c|c|c|c|}
\hline Age (years) & $49 \pm 7$ & $46 \pm 6$ & 0.15 \\
\hline Body mass index $\left(\mathrm{kg} / \mathrm{m}^{2}\right)$ & $24.2 \pm 4.4$ & $24.7 \pm 3.4$ & 0.6 \\
\hline Education (years) & $8.1 \pm 3.2$ & $8.7 \pm 3.4$ & 0.32 \\
\hline Social adjustment scale score & $1.7 \pm 0.4$ & $1.5 \pm 0.2$ & 0.12 \\
\hline Tobacco consumption (pack years of active smoking) & $34 \pm 16$ & $18 \pm 13$ & 0.09 \\
\hline Letter-number sequencing & $8.7 \pm 3.8$ & $11.3 \pm 3.1$ & 0.02 \\
\hline TMT-B (s) & $132 \pm 69.8$ & $80.3 \pm 43.2$ & 0.01 \\
\hline TMT-A (s) & $40.5 \pm 11.2$ & $31.2 \pm 7.6$ & 0.11 \\
\hline WAIS information & $8.3 \pm 3.6$ & $11.8 \pm 3.4$ & $<0.001$ \\
\hline
\end{tabular}

Abbreviations: AUDIT, Alcohol Use Disorders Identification Test; TMT-A, Trail-Making Test Part-A; TMT-B, Trail-Making Test Part-B; WAIS, Wechsler Adult Intelligence Third Revision.

Data are presented as mean \pm SD

*p-Values for between-group comparisons performed using two-sample t-test (demographic data) or Mann-Whitney U-test (neuropsychological tests). 
Table 2 Characteristics of the Alcohol-Dependent Subjects

\begin{tabular}{|c|c|c|}
\hline Patients' characteristics & Value & $\begin{array}{c}\text { Laboratory } \\
\text { norm }\end{array}$ \\
\hline Alcohol consumption $^{\text {a }}$ & $23.8 \pm 12.7$ & \\
\hline Duration of dependence (years) & $7.95 \pm 6.4$ & \\
\hline Abstinence (weeks) & $29 \pm 34$ & \\
\hline Age (years) at first drinking & $20.9 \pm 6.7$ & \\
\hline Age (years) at the onset of dependence & $39.8 \pm 10.6$ & \\
\hline \multicolumn{3}{|l|}{ Biological variables: } \\
\hline$\gamma$-Glutamyl-transferase & $53.16 \pm 6.98$ & $<53$ \\
\hline Alanine aminotransferase $(\mathrm{U} / \mathrm{l})$ & $26.53 \pm 15.42$ & $<38$ \\
\hline Aspartate aminotransferase (U/I) & $27.95 \pm 15.19$ & $<40$ \\
\hline $\begin{array}{l}\text { Aspartate aminotransferase/alanine } \\
\text { aminotransferase }\end{array}$ & $1.09 \pm 0.24$ & \\
\hline Carbohydrate-deficient transferrin & $1.97 \pm 0.25$ & $<2.6$ \\
\hline
\end{tabular}

${ }^{a}$ Consumption was defined as drinks per day during 3 months preceding detoxification, where one drink was considered to contain approximately $10 \mathrm{~g}$ of ethanol (standardization of beer, wine, and spirits).

Kaplan, 1979), and (3) a score $>20$ on Hamilton Anxiety Scale or a score $>10$ on Hamilton Depression Scale.

Healthy comparison subjects were recruited from the community. The inclusion criteria were alcohol consumption of less than two standard units (where one unit equals $10 \mathrm{~g}$ of ethanol) of alcohol per week and a score $\leqslant 5$ on the AUDIT scale.

All subjects underwent a thorough medical, psychiatric, and neurological examination by a senior psychiatrist. Exclusion criteria for all subjects included any kind of neurological symptoms (cerebellar, sensory, or motor dysfunctions), history of psychiatric disorder (other than alcohol-dependence for patients), medical conditions that may alter cerebral function (ie cardiovascular, endocrinological, autoimmune, or oncological diseases), and brain trauma (seizures, degenerative disease, previous head injury with loss of consciousness). In addition to these examinations, the presence of visible abnormalities on $\mathrm{T}_{2}$ images (assessed by a radiologist), pace maker, bypass surgery or metallic implants that would preclude magnetic resonance imaging (MRI) scan, or substance abuse (other than alcohol and tobacco) resulted to the exclusion of the subject.

Therefore, the set of exclusion criteria led up to select alcohol-dependent patients without apparent neurological conditions.

Subjects were fully informed of the nature of the investigation and they provided written consent for their involvement. The study was carried out in accordance with the Declaration of Helsinki. The study protocol was approved by the C.P.P. Île de France 7 ethics committee.

\section{Image Acquisition}

All subjects underwent volumetric MRI and DTI of the brain, using a $1.5 \mathrm{~T}$ Signa imager (General Electric Healthcare, Milwaukee, WI). Volumetric imaging was performed using a standard $3 \mathrm{D} \mathrm{T}_{1}$-weighted inversion recovery fast-spoiled gradient-recalled sequence with the following parameters: axial orientation, field of view $=24 \mathrm{~cm}$, matrix $=256 \times 192$ interpolated to $256 \times 256,124$ slice locations, $0.9375 \times$ $0.9375 \mathrm{~mm}^{2}$ in-plane resolution, slice thickness $=1.3 \mathrm{~mm}$, $\mathrm{TE}=2 \mathrm{~ms}, \mathrm{TR}=10 \mathrm{~ms}, \mathrm{TI}=600 \mathrm{~ms}$, flip angle $=10^{\circ}$, read bandwidth $=12.5 \mathrm{kHz}$.

Diffusion-weighted images were acquired with an echoplanar imaging sequence $(\mathrm{TE}=70 \mathrm{~ms}, \mathrm{TR}=8300 \mathrm{~ms}$, $b$-value $=700 \mathrm{~s} / \mathrm{mm}^{2}, 56$ slices of $2.4 \mathrm{~mm}$, axial orientation, in-plane resolution $=1.875 \times 1.875 \mathrm{~mm}$, matrix $128 \times 128$ ) . DTI acquisition included five supplementary $\mathrm{T}_{2}$ images without diffusion weighting $(b=0)$. Diffusion gradients were applied along 36 orientations. The acquisition process was designed to allow reconstruction of the diffusion tensor, even if subjects had moved during the scanning procedure (Dubois et al, 2006b). The number of orientations used to reconstruct the diffusion tensor was similar in the patient and control groups $(34.6 \pm 2.7$ and $35.3 \pm 1.9$, respectively; $t=-1.053, p=0.3)$.

\section{Data Processing and ROI Drawing for White Matter Tracking}

The tracking algorithm was initialized using a 'two-region of interest' approach (Conturo et al, 1999), where we focused on the fiber bundles passing through specific regions and connecting the midbrain to the pons and to the thalamus.

First, echo-planar distortion in the diffusion images was corrected, then diffusion data were processed and regions of interest (ROIs) were drawn using Brainvisa software (http:// brainvisa.info/). Thereafter, the diffusion tensor was assessed and the ADC and FA for each voxel were calculated on the basis of the diffusion tensor.

ROIs were manually defined by SC, who was blind to the subject's diagnosis, using the method described by Sullivan et al (2004). The pons and the thalamus (left and right separately) were traced in the sagittal plane with correction in the axial plane, whereas the midbrain was traced in an axial plane with correction in the sagittal plane. Each region was delineated using first a midplane, and then in five successive slices above and below that plane, or on the left and on the right from that plane, depending of its orientation. Then, corrections were made to ensure that two ROIs were separated from each other by a distance of at least five axial slices (Figure 1), as a result the midbrain was covered by seven successive axial slices. ROIs were drawn on individual $\mathrm{T}_{1}$ realigned anterior commissure-posterior commissure scans, rather than diffusion images, to avoid using the dependent variables (FA and ADC maps) for data preprocessing and analyses (Kanaan et al, 2006). An isotropic morphological erosion of $0.5 \mathrm{~mm}$ was applied to ROI masks, to prevent interference from other brainstem bundles in the tractography process. ROIs were then individually registered on diffusion images (t2-diffusion weighted, FA and ADC images) with an affine transformation minimizing mutual information implemented in Brainvisa software (Houenou et al, 2007; Dubois et al, 2006a). The locations of the transformed ROIs in native diffusion space were verified visually. 

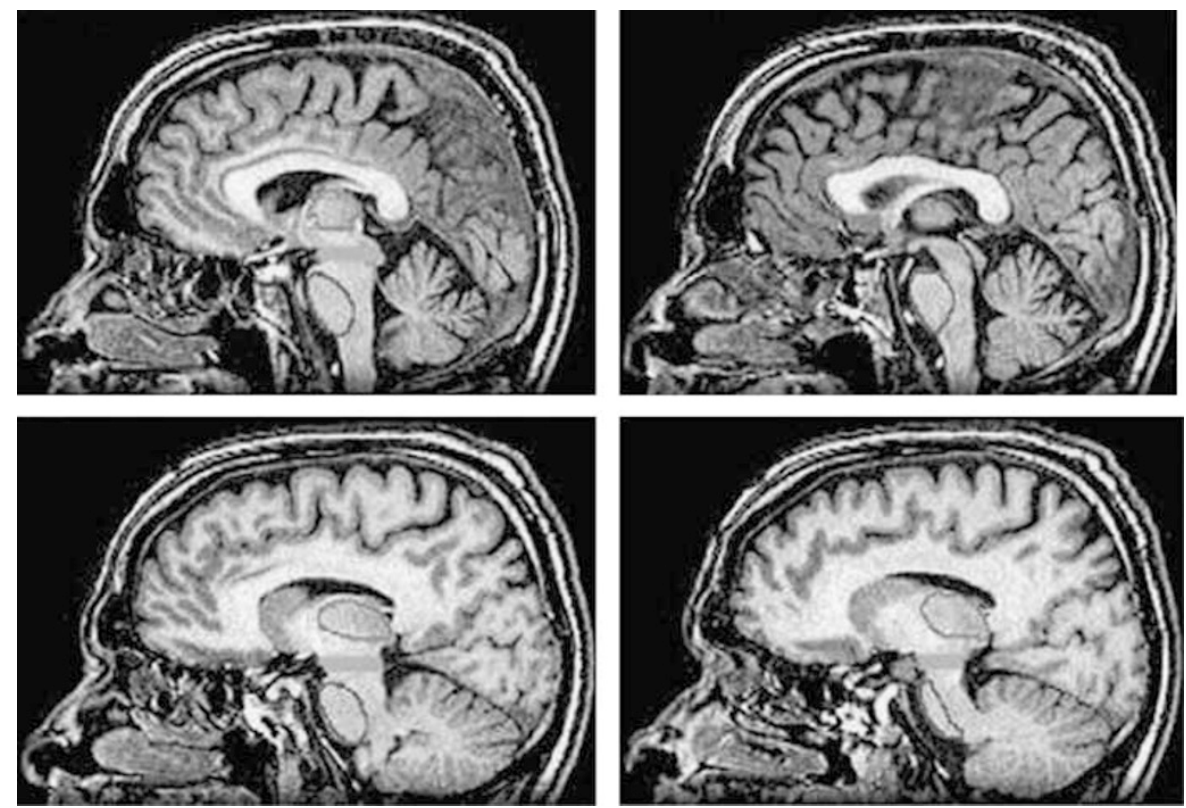

Figure I $T$, sagittal slices from a healthy man. Outlines, from the top to the bottom, of the thalamus, the midbrain and the pons.

\section{Bundle Tracking}

Tractography algorithms use DTI data for the virtual reconstruction of white matter fibers, by following the continuous path of greatest water diffusivity from a predefined brain region (ROI/seed points) through the white matter (Behrens et al, 2003; Conturo et al, 1999; Poupon et al, 2001).

The fiber components of bundles emerging from ROIs were reconstructed using a voxel-by-voxel regularized streamline algorithm (Perrin et al, 2005), which resembles diffusion tensor deflection (Lazar et al, 2003). This approach was supposed to minimize the disturbances caused by simple crossing configurations during tractography. The direction of the tract in each voxel was determined using two parameters: the largest tensor direction eigenvector (weighted by the coefficient $\alpha$ that corresponds to local anisotropy) and inertia (weighted by $1-\alpha$ ), ie the incident direction of the tract that represents the propagation direction from the previous reconstruction step. The algorithm proceeded toward the chosen direction (depending on the weighting and thus on local anisotropy) in halfvoxel steps. The propagation mask excluded voxels likely to belong to the gray matter $(\mathrm{FA}<0.2)$. A maximum curvature angle of $20^{\circ}$ was used to avoid high curvature of the trajectory. Only fibers passing from midbrain to pons or from midbrain to thalamus were analyzed.

For each reconstructed fiber bundle, we counted the number of tracked fibers and determined mean FA and mean ADC along these bundles. In this article, the term 'tracked fibers' refers to the number of fibers generated by the algorithm in fiber bundles connecting predefined brain regions, rather than the actual number of fibers in anatomical fiber bundles (for further explanation of this difference, see Discussion).

In order to avoid the possible confounding effect on tractography of interindividual differences in ROI volume, the number of fibers reconstructed between two ROIs were normalized by the volumes of both regions $(\mathrm{ROI} 1 \times \mathrm{ROI} 2$; cf Houenou et al, 2007).

\section{Neuropsychological Tests}

All participants underwent the Mini Mental State Examination (Folstein et al, 1975) and a battery of neuropsychological tests on the day of the MRI acquisition or within the following 3 days (Chanraud et al, 2007). We selected cognitive flexibility tasks in which alcohol-dependent subjects are known to perform worse than healthy subjects and which have been shown to be related to midbrain white matter volume (Chanraud et al, 2007): the TMT-B and the WAIS LNS (see Table 1). The TMT-B performance was measured as completion time and LNS performance as reproduced digits.

(1) We used the subjects' performance in a semantic memory task (WAIS-information) to evaluate the specificity of TMT-B and LNS to midbrain-pons/midbrainthalamus bundle characteristics, as the corticocerebellar circuit is not believed to be directly involved in semantic memory performance (Thompson-Schill, 2003).

(2) We also used the subjects' performance in the TMT-A in statistical analyses as a control for motor skills.

Raw tests scores were converted to $z$-scores by adjusting for age and years of education from the French population data.

\section{Statistical Analyses}

Statistical analyses were conducted with SPSS 12.0 software (SPSS Inc., Chicago, IL). The normality of the data was tested with Kolmogorov-Smirnov test. Differences between groups for demographic variables (Table 1) and ROI volumes were analyzed using $t$-tests for independent samples. 
In order to limit type 1 error (false positives), the number of variables examined was constrained by a priori hypotheses (detailed in Introduction) based on previous findings on the same samples (Chanraud et al, 2007), for both the subcortical regions and neuropsychological tests.

As the volumes of the pons and thalamus have been shown to be independently affected in alcohol-dependent subjects (Sullivan, 2003), we performed all statistical analyses separately for the bundles connecting the midbrain to the pons, and for those connecting the midbrain to the thalamus. Between-group differences in the number of tracked fibers/volume, FA, and ADC were assessed using the nonparametric rank-based Mann-Whitney $U$-test.

Associations between bundle features, neuropsychological $z$-scores, brain region volumes, and patients' drinking history variables were assessed with Pearson or Spearman correlations, as appropriate.

For each analysis, we fixed a two-tailed $\alpha$ level at 0.05 .

\section{RESULTS}

Drinking history variables for the alcohol-dependent subjects are presented in Table 2. On average, they had their first alcoholic drink in early adulthood and had been alcohol-dependent since approximately 40 years of age. The alcohol-dependent and control groups were similar with respect to mean age, years of education, body mass index, and Social Adjustment Scale Self Report and Mini Mental State Examination scores. At the time of MRI, a half of the alcoholics and $17 \%$ of the controls were cigarette smokers.

\section{Between-Group Comparisons}

In comparison with healthy subjects, the alcohol-dependent subjects performed worse on TMT-B, LNS, and WAISinformation but not on TMT-A (see Table 1).

Alcohol-dependent subjects had a smaller mean volume of the pons $\left(4784 \pm 1104\right.$ vs $5477 \pm 1074 \mathrm{~mm}^{3}$ in healthy subjects; $t(42)=-2.10 ; p=0.04)$ and there was also a nonsignificant trend toward a lower thalamus volume $\left(9330 \pm 1518\right.$ vs $\left.10070 \pm 1391 \mathrm{~mm}^{3} ; t(42)=-1.66 ; p=0.1\right)$. No difference was observed in midbrain volume (4363 \pm 496 vs $\left.4455 \pm 444 \mathrm{~mm}^{3} ; t(42)=-0.63 ; p=0.5\right)$.

The main results of bundle tracking are presented in Table 3. Compared to healthy subjects, alcohol-dependent subjects had, on average, $18 \%$ fewer fibers per unit volume running between the midbrain and the pons, and the mean $\mathrm{ADC}$ along the tracked fiber bundles between these two regions was 3\% higher. For the fiber bundles tracked between the midbrain and the thalamus, only ADC differed, being 3\% higher in alcohol-dependent subjects than in healthy subjects. A typical example of the tractography output in one alcohol-dependent subject is shown in Figure 2.

\section{Correlation Between Diffusion Indices in Tracked Bundles and Neuropsychological Data}

In the group of alcohol-dependent subjects, the normalized number of fibers tracked between the midbrain and the pons was related to the TMT-B $z$-scores $(r=-0.51 ; p=0.03$; see Figure 3a). The LNS $(r=0.19 ; p=0.41)$, the WAISinformation $(r=0.08 ; p=0.73)$, and the TMT-A $z$-scores $(r=-0.29 ; p=0.235$; see Figure $3 b)$ did not correlate with the characteristics of the midbrain-pons bundles. No correlation was found between the characteristics of the midbrain-thalamus bundles and neuropsychological data.

In the group of healthy subjects, there was no significant association between bundles' properties and TMT-B performance (see Figure 3a) or with other neuropsychological data.

\section{Correlation Between Diffusion Indices in Tracked Bundles, Neuropsychological Data, and Drinking History Variables}

Neither neuropsychological performance nor white matter values were related to the patients' drinking history

Table 3 Results of Bundle Tracking

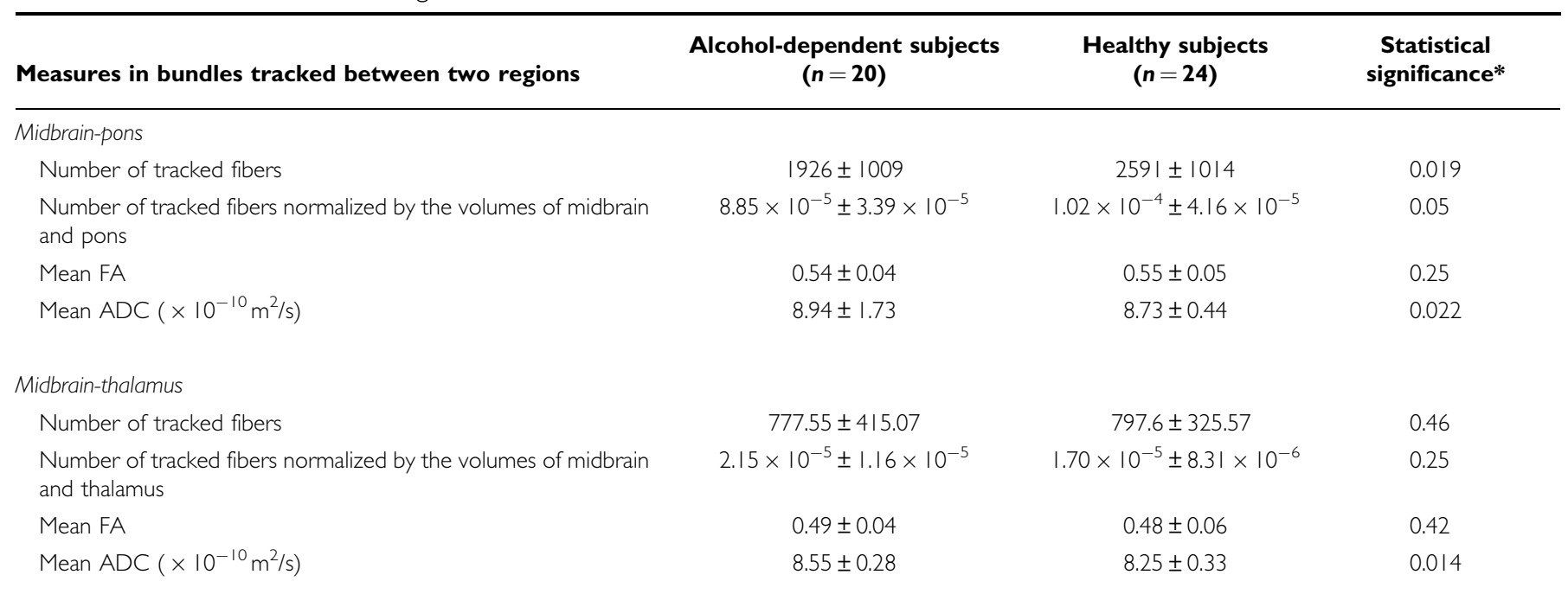

Abbreviations: ADC, apparent diffusion coefficient; FA, fractional anisotropy.

Data are presented as mean \pm SD.

*p-Values for between-group comparisons carried out with analysis of covariance (number of tracked fibers) or Mann-Whitney U-test (FA and ADC). 

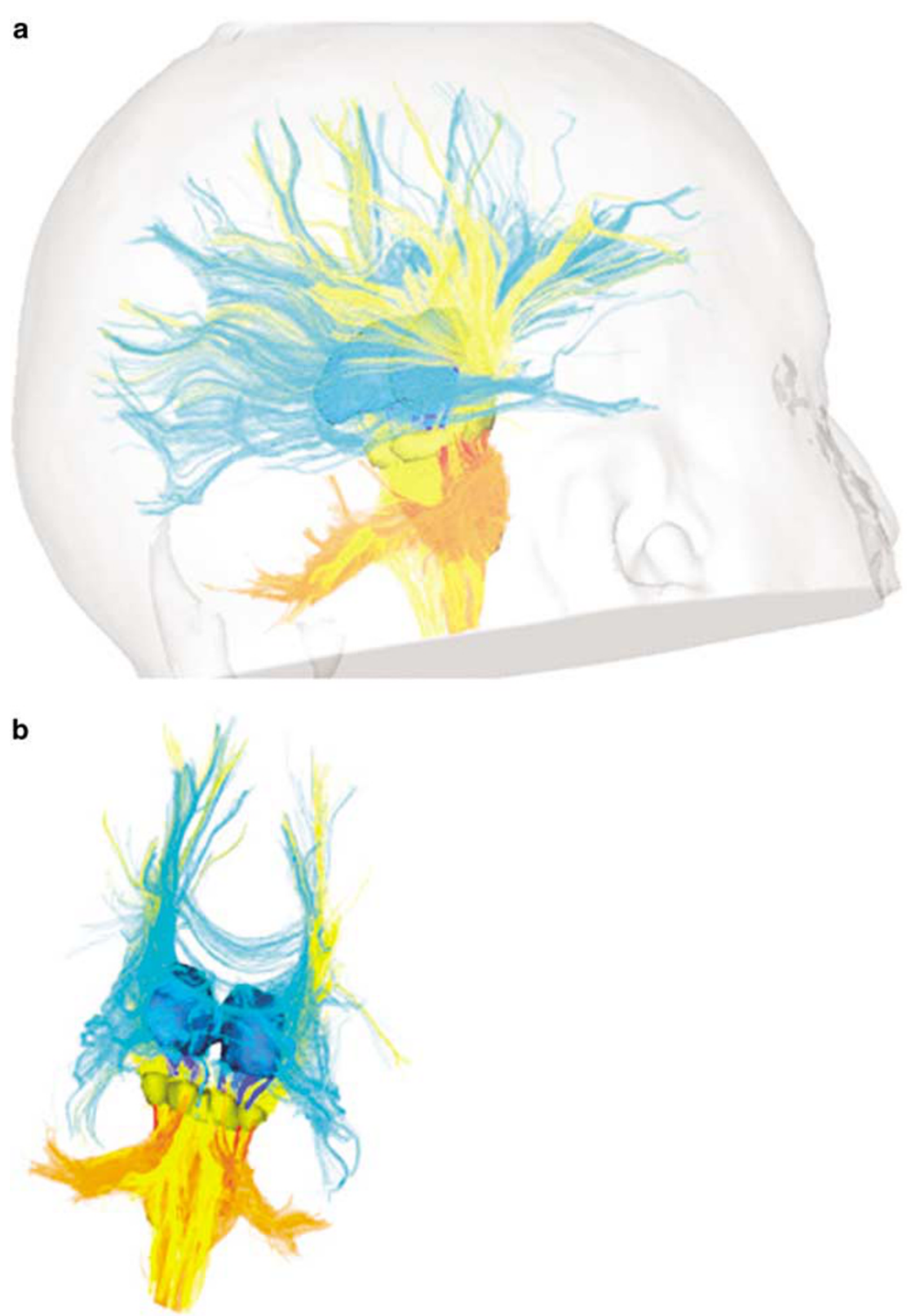

Figure 2 Tractography results in one healthy subject: (a) 3D view, (b) oblique plane. Orange: Pons and reconstructed fiber bundles connecting the pons and the cerebellum. Yellow: Midbrain and reconstructed fiber bundles passing through the midbrain. Light blue: Thalamus and reconstructed fiber bundles passing through the thalamus. Dark blue: Reconstructed fiber bundles connecting the midbrain to the thalamus. Red: Reconstructed fiber bundles connecting the midbrain to the pons.

variables such as: duration of dependence, duration of abstinence, age at first drinking, number of alcohol drinks per day, age of drinking onset, or AUDIT.

\section{DISCUSSION}

Alcohol-dependent subjects had fewer tracked white matter fibers per unit volume running between the midbrain and the pons, and a smaller pons volume compared to controls. Furthermore, the normalized number of such fibers was correlated with the alcohol-dependent subjects' performance in the neuropsychological TMT-B test. This is the first report of an alteration of the white matter microstructure between the midbrain and the pons that might have functional consequence for executive skills in alcoholdependent subjects without manifest neurological complications.

\section{Alcoholism-Associated Changes in White Matter Tracts}

Previously reported alcoholism-associated pathologic features include demyelination, loss of myelinated fibers and axonal deletion, which possibly results from regional neuronal loss (Harper et al, 2003). The reduction of the alcoholics' brain weight and volume, which is well documented in the literature (Harper and Matsumoto, 2005), is largely due to an overall decrease of white matter volume. According to our current data, moderately excessive use of alcohol may 

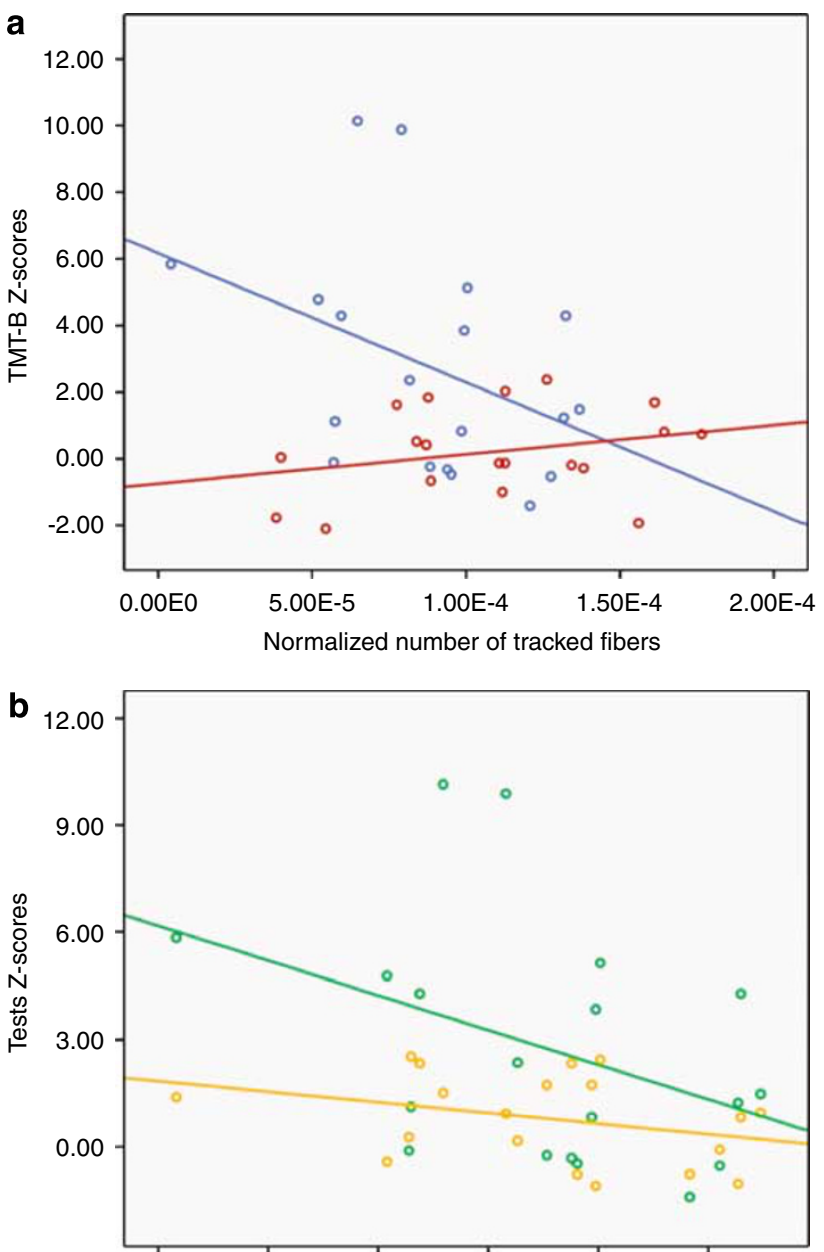

0.00E0 2.50E-5 5.00E-5 7.50E-5 1.00E-4 1.25E-4

Normalized number of tracked fibers

Figure 3 (a) The relationship between the normalized number of tracked midbrain-pons fibers and corrected $z$-scores obtained in the TrailMaking Test part-B (TMT-B) by the alcohol-dependent subjects (in blue; $r^{2}=-0.154$ ) and by the controls (in red; $r^{2}=-0.07$ I). (b) The relationship between the normalized number of tracked midbrain-pons fibers and corrected $z$-scores obtained by the alcohol-dependent subjects in the TMT-A (in orange; $r^{2}=-0.107$ ) and in TMT-B (in green; $r^{2}=-0.154$ ). In both graphs, high z-scores are in the direction of impairment.

also be associated with a loss of white matter fibers between the midbrain and the pons.

The abnormally high ADC along both midbrain-pons and midbrain-thalamus tracts suggest cell volume changes arising from tissue degeneration. More specifically, the degradation of cell membranes increases the extracellular space fraction, which allows larger net displacements of water molecules within a given diffusion time, and thus increases ADC (Sotak, 2004). As we did not study the whole brain, we cannot estimate whether this abnormally high ADC in alcoholics is specific to the explored regions, or more diffuse in nature.

Similar FA along the midbrain-pons tract in both alcohol-dependent and healthy subjects may be expected in a region, such as the pons, where FA reduction due to white matter fibers crossing may mask any reduction due to disease (Pierpaoli et al, 2001). Furthermore, the small change in ADC and the correlation between the number of tracked fibers and the pons volume are both consistent with a primary damage of the pons, which has caused secondary white matter degeneration (Pierpaoli et al, 2001). Although DTI cannot determine the nature of the neural alterations, the location of the detected abnormalities seems to imply a central pontine myelinolysis-like condition (Sullivan and Pfefferbaum, 2001). As already mentioned by Sullivan and Pfefferbaum (2001), this condition may occur with higher incidence in subjects without gross neurological deficits and contribute to their neuropsychological impairment.

\section{Correlation with Neuropsychological Performance}

TMT-B evaluates several different processes, such as 'cognitive flexibility' and 'set shifting', as well as 'general attention' (Zakzanis et al, 2005). The correlation between the normalized number of tracked midbrain-pons fibers and TMT-B score supports the assumption that microstructural white matter alterations may contribute to the neuropsychological impairment observed in alcohol-dependent subjects. Taken together, our results indicate that fiber bundles linking the midbrain and the pons might be related to executive skills (Schmahmann et al, 2004). This interpretation is also in line with our previous finding of the association between midbrain volume and cognitive flexibility in alcohol-dependent subjects (Chanraud et al, 2007).

In contrast with TMT-B, we observed no statistically significant correlation between LNS scores and the normalized number of tracked midbrain-pons fibers. One possible explanation for this difference could be that the TMT-B task incorporates an additional motor component, whereas LNS focuses more purely on the use of working memory. It should be noted that the cognitive flexibility deficits of our alcohol-dependent study subjects cannot be explained by markedly impaired motor processing, because they were no slower than control subjects in TMT-A. Our current results thus lend support to the proposition that there are separate pathways within the corticocerebellar circuit underlying the cognitive and motor processes (Kelly and Strick, 2003; Schmahmann, 2004), which are, however, strongly interconnected.

Our findings, together with those of Sullivan (2003), suggest that some cognitive process impairment in alcoholdependent subjects could be linked not only to cortical dysfunction but also to modulatory influences from subcortical structures. Alcohol-associated alterations in the corticocerebellar network may have a bottleneck effect on central decision-making processes (Sigman and Dehaene, 2005). In other words, a part of the executive dysfunction in alcohol-dependent subjects could possibly be attributed either to less cortical information or to fewer modulatory signals from subcortical structures arriving at the cerebellum. In addition to cognitive impairment, the disruption of selective neural circuits may also contribute to the complex neurologic symptomatology observed in chronic alcoholism (Koob, 2003). However, it must be emphasized that because our study had a cross-sectional design, we cannot ascribe a causal interpretation of the findings. The structural abnormalities that we observed in our alcohol-dependent subjects may result from long-term alcohol abuse/dependence, or they may reflect a preexisting factor that had 
predisposed these subjects to severe alcoholism. In both instances, these neuroanatomic changes may contribute to explain why cognitive behavioral therapy often has a rather limited effect in these patients (Morgenstern and Longabaugh, 2000). Indeed, this kind of therapy is based on changing behaviors in spite of the fact that cognitive impairment limits experience-dependent recovery (Roehrich and Goldman, 1993). Our findings further support the hypothesis that neuroanatomical changes underlying such cognitive impairment could require establishment of compensatory mechanisms (Desmond et al, 2003; Pfefferbaum et al, 2001).

\section{Methodological Considerations}

We used a quantitative fiber-tracking approach to analyze FA, ADC, and the number of tracked fibers per unit volume within the bundles of interest. Fibers reconstructed by tractography algorithms cannot be seen as one-to-one correlates of existing anatomical, histologically determined fiber bundles. Instead, tractography gives a statistical representation of both the voxel-to-voxel coherence of brain matter and the connectivity of white matter tracts, which is, nonetheless, highly representative of the underlying anatomy (Sullivan et al, 2006). Tracked fibers were classified using an anatomical white matter atlas (Nieuwenhuys et al, 1988) and previous tractography studies (Hagmann et al, 2003; Stieltjes et al, 2001). The fibers that we tracked between the midbrain and the pons are believed to represent the corticopontine tract, the ascending cholinergic reticular tract (Nieuwenhuys et al, 1988), and probably parts of the corticospinal and corticobulbar tracts (Hagmann et al, 2003; Stieltjes et al, 2001). Similarly, the fibers we tracked between the midbrain and thalamus are believed to represent the superior cerebellar peduncle, the medial lemniscus (Stieltjes et al, 2001), and the ascending cholinergic reticular tract.

\section{Limitations}

The main limitation of this study arises from the already discussed fact that the number of virtual, reconstructed fibers cannot be directly interpreted as anatomical correlate of real fibers in the respective bundle. The DTI tractography results may be biased by many unspecific factors (JohansenBerg and Behrens, 2006). Different macro- and microstructural characteristics of fiber bundles, such as myelination, axonal and glial loss, shape, width and curvature of the fiber tract, tissue hydration, and overall brain size can influence the functioning of tractography algorithms (Catani, 2006; Catani et al, 2003; Pierpaoli et al, 2001). Nevertheless, previous studies have shown that the reconstructed fiber bundle metrics can provide a sufficiently good surrogate marker of real fiber anatomy (Sullivan et al, 2006). Our present results are also supported by earlier post-mortem studies, which have demonstrated a loss of white matter fibers - especially those of noradrenergic (Arango et al, 1994) and serotoninergic (Halliday et al, 1993) neurons - in the brainstem of alcohol-dependent subjects.

Another limitation stems from tobacco use in the alcoholdependent population, as half of the alcohol-dependent subjects included in this study were smokers. Neuropsychological performance and brain structural differences were reported between smoking and nonsmoking abstinent alcohol-dependent subjects (Meyerhoff et al, 2006). However, the interaction of smoking and chronic alcoholism is still unclear. Furthermore, statistical analyses between smoking and nonsmoking alcohol-dependent subjects found no difference in performance or white matter values.

Then, as mentioned above, causal inferences from our data are not possible because of the cross-sectional design.

Finally, our findings and interpretations are drawn from a sample of men. As alcohol-related neuropsychological deficits and brain alterations are gender dependent (Oscar-Berman et al, 1997), the questions raised in this study still remain for alcoholic women.

\section{Conclusion}

By applying a quantitative fiber-tracking technique to DTI data, we were able to demonstrate in detoxified and apparently neurologically intact alcohol-dependent subjects, marked alterations in mesencephalic white matter bundles, which may underlie their cognitive flexibility impairment. These findings suggest that an early assessment of white matter microstructure and cognitive measures of mental flexibility may provide major insights into capacity to benefit from a heavy emphasis on educational methods early in treatment, even if a patient has a high level of education or intellectual ability. We speculate that future longitudinal DTI studies of abstinent alcoholics might specify which constituents of white matter microstructure exhibit recovery and could thus contribute to restitution of function. Further, considering a link between the microstructural integrity of white matter tracts and neuropsychological performance impairment, the degree of anatomical recovery in this brain region could possibly be estimated with standard neuropsychological tests.

\section{ACKNOWLEDGEMENTS}

We thank Professor André Syrota and Dr Bertrand Nalpas for their support, and Dr Margaret Rosenbloom and Andrew Rhein for helpful comments on the article.

\section{FINANCIAL DISCLOSURES}

The study was supported by grants from the French Society of Alcohology (Société Française d'Alcoologie, SFA), the French Interministry Mission Against Drugs and Addiction (Mission Interministérielle de Lutte contre la Drogue et la Toxicomanie, MILDT, No A05248LS), the INSERM ATC Alcool 2007, and the INSERM PNR Alcool 2008. Dr J Penttilä has received personal grants from the Finnish Cultural Foundation and the Sigrid Jusélius Foundation, Finland.

The authors have no financial interests or potential conflicts of interest.

\section{REFERENCES}

Annett M (1967). The binomial distribution of right, mixed and left handedness. Q J Exp Psychol 19: 327-333. 
Arango V, Underwood MD, Mann JJ (1994). Fewer pigmented neurons in the locus coeruleus of uncomplicated alcoholics. Brain Res 650: 1-8.

Arria AM, Tarter RE, Starzl TE, Van Thiel DH (1991). Improvement in cognitive functioning of alcoholics following orthotopic liver transplantation. Alcohol Clin Exp Res 15: 956-962.

Baker KG, Harding AJ, Halliday GM, Kril JJ, Harper CG (1999). Neuronal loss in functional zones of the cerebellum of chronic alcoholics with and without Wernicke's encephalopathy. Neuroscience 91: 429-438.

Behrens TE, Johansen-Berg H, Woolrich MW, Smith SM, WheelerKingshott CA, Boulby PA et al (2003). Non-invasive mapping of connections between human thalamus and cortex using diffusion imaging. Nat Neurosci 6: 750-757.

Catani M (2006). Diffusion tensor magnetic resonance imaging tractography in cognitive disorders. Curr Opin Neurol 19: 599-606.

Catani M, Jones DK, Donato R, Ffytche DH (2003). Occipitotemporal connections in the human brain. Brain 126(Part 9): 2093-2107.

Chanraud S, Martelli C, Delain F, Kostogianni N, Douaud G, Aubin $\mathrm{HJ}$ et al (2007). Brain morphometry and cognitive performance in detoxified alcohol-dependents with preserved psychosocial functioning. Neuropsychopharmacology 32: 429-438.

Cohen JA, Kaplan MM (1979). The SGOT/SGPT ratio-an indicator of alcoholic liver disease. Dig Dis Sci 24: 835-838.

Conturo TE, Lori NF, Cull TS, Akbudak E, Snyder AZ, Shimony JS et al (1999). Tracking neuronal fiber pathways in the living human brain. Proc Natl Acad Sci USA 96: 10422-10427.

Desmond JE, Chen SH, DeRosa E, Pryor MR, Pfefferbaum A, Sullivan EV (2003). Increased frontocerebellar activation in alcoholics during verbal working memory: an fMRI study. Neuroimage 19: 1510-1520.

Dubois J, Hertz-Pannier L, Dehaene-Lambertz G, Cointepas Y, Le Bihan D (2006a). Assessment of the early organization and maturation of infants' cerebral white matter fiber bundles: a feasibility study using quantitative diffusion tensor imaging and tractography. Neuroimage 30: 1121-1132.

Dubois J, Poupon C, Lethimonnier F, Le Bihan D (2006b). Optimized diffusion gradient orientation schemes for corrupted clinical DTI data sets. Magma 19: 134-143.

Duka T, Townshend JM, Collier K, Stephens DN (2003). Impairment in cognitive functions after multiple detoxifications in alcoholic inpatients. Alcohol Clin Exp Res 27: 1563-1572.

Folstein MF, Folstein SE, McHugh PR (1975). 'Mini-mental state'. A practical method for grading the cognitive state of patients for the clinician. J Psychiatr Res 12: 189-198.

Goldman MS (1990). Experience-dependent neuropsychological recovery and the treatment of chronic alcoholism. Neuropsychol Rev 1: 75-101.

Hagmann P, Thiran JP, Jonasson L, Vandergheynst P, Clarke S, Maeder $\mathrm{P}$ et al (2003). DTI mapping of human brain connectivity: statistical fibre tracking and virtual dissection. Neuroimage 19: 545-554.

Halliday G, Ellis J, Heard R, Caine D, Harper C (1993). Brainstem serotonergic neurons in chronic alcoholics with and without the memory impairment of Korsakoff's psychosis. J Neuropathol Exp Neurol 52: 567-579.

Harper C, Dixon G, Sheedy D, Garrick T (2003). Neuropathological alterations in alcoholic brains. Studies arising from the New South Wales Tissue Resource Centre. Prog Neuropsychopharmacol Biol Psychiatry 27: 951-961.

Harper C, Matsumoto I (2005). Ethanol and brain damage. Curr Opin Pharmacol 5: 73-78.

Houenou J, Wessa M, Douaud G, Leboyer M, Chanraud S, Perrin M et al (2007). Increased white matter connectivity in euthymic bipolar patients: diffusion tensor tractography between the subgenual cingulate and the amygdalo-hippocampal complex. Mol Psychiatry 1: 1.
Hyman SE, Malenka RC, Nestler EJ (2006). Neural mechanisms of addiction: the role of reward-related learning and memory. Annu Rev Neurosci 29: 565-598.

Johansen-Berg H, Behrens TE (2006). Just pretty pictures? What diffusion tractography can add in clinical neuroscience. Curr Opin Neurol 19: 379-385.

Kanaan RA, Shergill SS, Barker GJ, Catani M, Ng VW, Howard R et al (2006). Tract-specific anisotropy measurements in diffusion tensor imaging. Psychiatry Res 146: 73-82.

Kelly RM, Strick PL (2003). Cerebellar loops with motor cortex and prefrontal cortex of a nonhuman primate. J Neurosci 23: 8432-8444.

Koob GF (2003). Alcoholism: allostasis and beyond. Alcohol Clin Exp Res 27: 232-243.

Kril JJ, Halliday GM, Svoboda MD, Cartwright H (1997). The cerebral cortex is damaged in chronic alcoholics. Neuroscience 79: 983-998.

Lazar M, Weinstein DM, Tsuruda JS, Hasan KM, Arfanakis K, Meyerand ME et al (2003). White matter tractography using diffusion tensor deflection. Hum Brain Mapp 18: 306-321.

Le Bihan D, Mangin JF, Poupon C, Clark CA, Pappata S, Molko N et al (2001). Diffusion tensor imaging: concepts and applications. J Magn Reson Imaging 13: 534-546.

Meyerhoff DJ, Tizabi Y, Staley JK, Durazzo TC, Glass JM, Nixon SJ (2006). Smoking comorbidity in alcoholism: neurobiological and neurocognitive consequences. Alcohol Clin Exp Res 30: 253-264.

Morgenstern J, Longabaugh R (2000). Cognitive-behavioral treatment for alcohol dependence: a review of evidence for its hypothesized mechanisms of action. Addiction 95: $1475-1490$.

Nieuwenhuys R, Voogd J, vanHuijzen C (1988). The Human Central Nervous System. A Synopsis and Atlas, 3rd rev edn. Springer-Verlag: Berlin.

Oscar-Berman M, Shagrin B, Evert DL, Epstein C (1997). Impairments of brain and behavior: the neurological effects of alcohol. Alcohol Health Res World 21: 65-75.

Perrin M, Poupon C, Rieul B, Leroux P, Constantinesco A, Mangin JF et al (2005). Validation of q-ball imaging with a diffusion fibre-crossing phantom on a clinical scanner. Philos Trans $R$ Soc Lond B Biol Sci 360: 881-891.

Pfefferbaum A, Adalsteinsson E, Sullivan EV (2006). Supratentorial profile of white matter microstructural integrity in recovering alcoholic men and women. Biol Psychiatry 59: 364-372.

Pfefferbaum A, Desmond JE, Galloway C, Menon V, Glover GH, Sullivan EV (2001). Reorganization of frontal systems used by alcoholics for spatial working memory: an fMRI study. Neuroimage 14: 7-20.

Pfefferbaum A, Sullivan EV, Hedehus M, Adalsteinsson E, Lim KO, Moseley M (2000). In vivo detection and functional correlates of white matter microstructural disruption in chronic alcoholism. Alcohol Clin Exp Res 24: 1214-1221.

Pierpaoli C, Barnett A, Pajevic S, Chen R, Penix LR, Virta A et al (2001). Water diffusion changes in Wallerian degeneration and their dependence on white matter architecture. Neuroimage 13(6 Part 1): 1174-1185.

Poupon C, Mangin J, Clark CA, Frouin V, Regis J, Le Bihan D et al (2001). Towards inference of human brain connectivity from MR diffusion tensor data. Med Image Anal 5: 1-15.

Reinert DF, Allen JP (2002). The Alcohol Use Disorders Identification Test (AUDIT): a review of recent research. Alcohol Clin Exp Res 26: 272-279.

Roehrich L, Goldman MS (1993). Experience-dependent neuropsychological recovery and the treatment of alcoholism. J Consult Clin Psychol 61: 812-821.

Schmahmann JD (2004). Disorders of the cerebellum: ataxia, dysmetria of thought, and the cerebellar cognitive affective syndrome. J Neuropsychiatry Clin Neurosci 16: 367-378. 
Schmahmann JD, Ko R, MacMore J (2004). The human basis pontis: motor syndromes and topographic organization. Brain 127(Part 6): 1269-1291.

Schmahmann JD, Pandya DN (1997). The cerebrocerebellar system. Int Rev Neurobiol 41: 31-60.

Schulte T, Muller-Oehring EM, Salo R, Pfefferbaum A, Sullivan EV (2006). Callosal involvement in a lateralized stroop task in alcoholic and healthy subjects. Neuropsychology 20: 727-736.

Sigman M, Dehaene S (2005). Parsing a cognitive task: a characterization of the mind's bottleneck. PLoS Biol 3: e37.

Sotak CH (2004). Nuclear magnetic resonance (NMR) measurement of the apparent diffusion coefficient (ADC) of tissue water and its relationship to cell volume changes in pathological states. Neurochem Int 45: 569-582.

Stieltjes B, Kaufmann WE, van Zijl PC, Fredericksen K, Pearlson GD, Solaiyappan $\mathrm{M}$ et al (2001). Diffusion tensor imaging and axonal tracking in the human brainstem. Neuroimage 14: 723-735.

Sullivan EV (2003). Compromised pontocerebellar and cerebellothalamocortical systems: speculations on their contributions to cognitive and motor impairment in nonamnesic alcoholism. Alcohol Clin Exp Res 27: 1409-1419.

Sullivan EV, Adalsteinsson E, Pfefferbaum A (2006). Selective agerelated degradation of anterior callosal fiber bundles quantified in vivo with fiber tracking. Cereb Cortex 16: 1030-1039.
Sullivan EV, Deshmukh A, Desmond JE, Lim KO, Pfefferbaum A (2000). Cerebellar volume decline in normal aging, alcoholism, and Korsakoff's syndrome: relation to ataxia. Neuropsychology 14: 341-352.

Sullivan EV, Pfefferbaum A (2001). Magnetic resonance relaxometry reveals central pontine abnormalities in clinically asymptomatic alcoholic men. Alcohol Clin Exp Res 25: 1206-1212.

Sullivan EV, Rosenbloom M, Serventi KL, Pfefferbaum A (2004). Effects of age and sex on volumes of the thalamus, pons, and cortex. Neurobiol Aging 25: 185-192.

Sullivan EV, Rosenbloom MJ, Serventi KL, Deshmukh A, Pfefferbaum A (2003). Effects of alcohol dependence comorbidity and antipsychotic medication on volumes of the thalamus and pons in schizophrenia. Am J Psychiatry 160: 1110-1116.

Thompson-Schill SL (2003). Neuroimaging studies of semantic memory: inferring 'how' from 'where'. Neuropsychologia 41: 280-292.

Wakana S, Jiang H, Nagae-Poetscher LM, van Zijl PC, Mori S (2004). Fiber tract-based atlas of human white matter anatomy. Radiology 230: 77-87.

Weissman MM, Bothwell S (1976). Assessment of social adjustment by patient self-report. Arch Gen Psychiatry 33: 1111-1115.

Zakzanis KK, Mraz R, Graham SJ (2005). An fMRI study of the Trail Making Test. Neuropsychologia 43: 1878-1886. 